\title{
Migrazione e salute mentale: un problema emergente
}

\author{
Luca Cimino
}

\section{Riassunto}

La salute mentale dei migranti è da considerarsi oggi in Italia e in Europa uno dei maggiori problemi di salute individuale e pubblica.

La sempre maggiore presenza dei migranti che accedono ai servizi di salute mentale ha evidenziato non solo nuove e peculiari espressioni di disagio psichico legate alle specifiche dimensioni culturali ed etniche di ogni singola popolazione, ma soprattutto che i migranti rappresentano una popolazione piuttosto fragile e a rischio di sviluppare disturbi mentali.

Le evidenze scientifiche accumulate negli ultimi anni sull'aumentato rischio nei migranti di disturbi mentali gravi, quali le psicosi, hanno contribuito a determinare una "revisione generale" dei modelli interpretativi della genesi dei disturbi psichici poiché hanno rilevato l'importanza dei fattori di stress ambientale sullo sviluppo di tali disturbi, evidenziando che le caratteristiche culturali e la storia migratoria sono in grado di influenzare fortemente non solo il rischio di ammalarsi, ma anche la risposta alle cure.

\section{Résumé}

La santé mentale des migrants est aujourd'hui l'un des plus gros problèmes de santé individuelle et publique en Italie et en Europe.

Le nombre croissant de migrants qui accèdent aux services de santé mentale a mis en évidence non seulement de nouvelles typologies de la détresse psychique liées à des dimensions culturelles et ethniques de chaque population, mais aussi le fait que les immigrés courent un risque plus élevé que le reste de la population de développer des troubles mentaux.

Les données scientifiques accumulées depuis quelques années sur le risque augmenté des migrants de développer des maladies mentales graves, comme la psychose, ont conduit à une révision générale des modèles interprétatifs sur la genèse des troubles psychiques, soulignant l'importance des facteurs environnementaux non seulement pour le développement de ces troubles, mais aussi pour la réponse aux traitements.

\section{Abstract}

Mental health of migrants is nowadays one of the greatest individual and public health problems Italy and Europe are facing.

The increasing number of migrants who access mental health services shows not only new typologies of psychic distress linked to specific cultural and ethnic dimensions of every population, but also the fact that migrants are a fragile group at risk of developing mental disorders.

Scientific evidence accumulated in the past years about migrants' increased risk of developing severe mental illnesses, such as psychosis, lead to a general review of interpretative models about the genesis of mental illness. This is because these models noted the importance of environmental stressors on the development of these disorders, showing that cultural characteristics and migrant backgrounds can have a big impact not only on the risk of developing a mental disorder, but also the response to treatments.

Key words: migration; mental health; environmental stressors; cultural characteristics; migrant backgrounds.

"Se si guarda all'economia globale dal punto di vista della gente, il suo più grande fallimento consiste nell'incapacità di cercare lavoro sufficiente nei luoghi in cui le persone vivono"

Juan Somavia, direttore generale ILO (Organizzazione Internazionale del lavoro"

\footnotetext{
- Psichiatra, psicoterapeuta, medico legale, criminologo e psichiatra forense - Università di Bologna.
} 


\section{Introduzione.}

La salute mentale dei migranti è da considerarsi oggi in Italia e in Europa uno dei maggiori problemi di salute individuale e pubblica. A differenza di altri paesi europei, come Inghilterra e Francia, dove la migrazione legata ad un passato coloniale è una componente strutturale della società, l'Italia, invece, si è trovata a confrontarsi solo di recente con una tumultuosa trasformazione identitaria, divenendo da paese di emigrazione a paese di immigrazione; infatti l'aumento degli stranieri residenti nel nostro Paese è stato di circa 3 milioni di unità nel corso dell'ultimo decennio e di quasi 1 milione nell'ultimo biennio $^{1}$. In Italia, in Europa e nel resto del mondo il fenomeno migratorio ha mostrato una crescita rapida ed esponenziale, rilevando come i migranti presentino ingenti problematiche di salute fisica e mentale 2 .

La sempre maggiore presenza dei migranti che accedono ai servizi di salute mentale ha evidenziato non solo nuove e peculiari espressioni di disagio psichico legate alle specifiche dimensioni culturali ed etniche di ogni singola popolazione, ma soprattutto che i migranti rappresentano una popolazione piuttosto fragile e a rischio di sviluppare disturbi mentali; infatti è noto da tempo che la morbilità psichiatrica in tale tipologia di soggetti è maggiore che nei nativi, soprattutto per i disturbi mentali gravi ${ }^{3}$.

1 Caritas/Migrantes, Immigrazione: Dossier Statistico 2010, Edizioni IDOS, Roma, 2010.

${ }^{2}$ Bria P., Caroppo E., Salute mentale, migrazione e pluralismo culturale, Alpes Italia srl, Roma, 2008.

3 Tarricone I., Stivanello E., Ferrari S., Colombini N., Bolla E., Braca M., Giubbarelli C., Costantini C., Cazzamalli S., Mimmi S., Tedesco D., Menchetti M., Rigatelli M., Maso E., Balestrieri M., Vender S., Berardi D., "Migrant pathways to community mental health centres in Italy", International Journal of Social Psychiatry, 58, 2012, pp. 505-511; Braca M., Berardi D., Mencacci E., Murri M.B., Mimmi S., Allegri F., Mazzi F., Menchetti
Le evidenze scientifiche accumulate negli ultimi anni sull'aumentato rischio nei migranti di disturbi mentali gravi, quali le psicosi, hanno contribuito a determinare una "revisione generale" dei modelli interpretativi della genesi dei disturbi psichici poiché hanno rilevato l'importanza dei fattori di stress ambientale, non solo individuali, ma anche di area, sullo sviluppo di tali disturbi, evidenziando che le caratteristiche culturali e la storia migratoria sono in grado di influenzare fortemente non solo il rischio di ammalarsi, ma anche la risposta alle cure ${ }^{4}$.

\section{La migrazione come fattore di rischio di disturbo mentale.}

L'immigrazione è un processo fonte di notevole stress, tanto che una vasta letteratura in merito è ormai concorde nel rilevare che la presenza di questo dato nell'anamnesi personale o familiare costituisce un fattore di rischio per lo sviluppo di patologie mentali, prevalentemente appartenenti allo spettro schizofrenico ${ }^{5}$.

La difficoltà di superare le barriere culturali e linguistiche, lo sradicamento fisico e culturale dal proprio paese di origine, la lontananza dalla propria

M., Tarricone I., "Understanding psychopathology in migrants: A mixed categoricaldimensional approach", International Journal of Social Psychiatry, 2, June 2013.

${ }^{4}$ Boydell J., van Os J., Mckenzie K. et al., "Incidence of schizophrenia in ethnic minorities in London: ecological study into interactions with environment", British Medical Journal, 323, 2001, pp. 1336-1338; Tarricone I., Cianconi P., Chierzi F. et al., "Comprendere le cause dell'esordio psicotico dall'ascolto della storia migratoria: il lavoro del Bologna Transcultural Psychiatric Team (BOTPT)", in Popolo R., Balbi A., Vinci G., Interventi precoci nelle psicosi, Alpes Italia srl, Roma 2013.

${ }^{5}$ Harrison G., Holton A., Neilson D., Owens D., Boot D., Cooper J., "Severe mental disorder in Afro-Caribbean patients: some social, demographic and service factors", Psychological Medicine, 19(3), 1989, pp. 683-696; Morgan C., Charalambides M., Hutchinson G., Murray RM., "Migration, ethnicity, and psychosis: toward a socio developmental model", Schizophrenia Bulletin 36, 4, 2010, pp. 655-664. 
struttura familiare e amicale, la possibilità di essere esposti a situazioni estremamente a rischio come la prostituzione, la detenzione, la dipendenza, rappresentano una sorta di "rivoluzione" dell'identità, dei ruoli, dei codici, delle scelte di vita, che facilitano lo "scivolamento" verso varie forme di disagio psichico, spesso gravi ${ }^{6}$.

La condizione psicologica del "migrante recente", caratterizzata da una situazione del "nessun dove", dell'etichettamento, dell'incertezza del proprio stare nel mondo, dalla perdita della traccia di sé, da biografie al confine e da vissuti persecutori ${ }^{7}$, ovvero da fattori "squassanti" quei pilastri definitori dell'identità quali l'ipseità e l'idemità, concorre ad accrescere il rischio dell'insorgenza di disturbi mentali gravi, soprattutto psicotici.

La separazione rispetto al contesto familiare, affettivo, sociale e culturale originario, la partenza, il viaggio, l'arrivo e l'incognito, accompagnandosi a vissuti di profonda ansia nel migrante, producono la rottura di equilibri prestabiliti. L'emigrante si trova di fronte alla sfida di dover ridefinire il proprio progetto di vita, di delinearne le coordinate nello spazio, nel tempo e anche nel corpo, laddove il "corpo vissuto" del migrante rispecchia il "corpo percepito" dalla società occidentale, che spesso rimanda ad una immagine collettiva negativa di "bruttezza" e di alienazione che alimenta nel soggetto un enorme complesso di inferiorità che annulla la capacità di decidere e di essere se stesso $^{8}$. La "traiettoria" del migrante non è solo spaziotemporale, ma anche mentale ed emotiva; le aspettative e le condizioni dell'arrivo possono condizionare tutto il percorso futuro in quanto è in questo contesto che si gioca il passaggio

\footnotetext{
${ }^{6}$ Bria P., Caroppo E., Salute mentale, migrazione e pluralismo culturale, Alpes Italia srl, Roma, 2008.

${ }^{7}$ Sossi F., Migrare, Il Saggiatore, Roma, 2007.

8 Merleau-Ponty M., Fenomenologia delle percezione, Bompiani, Torino 2003.
}

"dell'illusione dell'emigrazione" alla "sofferenza dell'immigrazione", ovvero la possibilità che il migrante ha di ridefinirsi come progetto di nuova vita.

L'esperienza migratoria viene a rappresentare uno choc culturale ed identitario in cui il soggetto si trova di fronte alla sfida di dover ridefinire il proprio progetto di vita, di delinearne le coordinate nello spazio e nel tempo; si tratta di una vera e propria rottura traumatica della continuità narrativa biografica che il migrante deve riuscire a ricomporre, ricreando una unità narrativa intellegibile, attraverso l'elaborazione del lutto della separazione dal gruppo originario, dai legami costituiti durante l'infanzia e interiorizzati nella sua costruzione psico-affettiva, pena lo smarrimento identitario ed anche il rischio di caduta nell'abisso psicotico quale estremo tentativo di dare senso ad un'esperienza alienante. Il trovarsi in un sistema di relazioni che il soggetto migrante non riesce ad interpretare e che lo vive come corpo estraneo, l'assenza di una rete famigliare di supporto, possono alimentare vissuti di inferiorità sociale e di minorazione culturale che finiscono per tradursi in quella "estrema solitudine" in cui la persona finisce per diventare estranea a se stessa generando varie forme di disagio psichico. Il non-esserci-nel-mondoe-col-mondo finisce per generare processi di alienazione e di depersonalizzazione che si traducono in quella "crisi della presenza" del Dasain, che si manifesta attraverso il declinarsi psicopatologico delle condizioni ontologiche dell'esistenza quali il temporalizzarsi, lo spazializzarsi, il coesistere ed il progettarsi, e che finisce per concretizzarsi in una modalità psicotica di "essere-nel-mondo".

Gli studi epidemiologici hanno, infatti, rilevato un "eccesso di psicosi" tra i migranti delle prime e delle 
seconde generazioni ${ }^{9}$, ma non una maggiore prevalenza di Disturbi Mentali Comuni (DMC), quali ansia e depressione, tra i migranti rispetto ai nativi ${ }^{10}$. Possibili fattori di rischio per l'insorgenza di disturbi depressivi e d'ansia nei migranti e nelle minoranze etniche sono rappresentati da una storia migratoria traumatica, da un maggiore carico familiare e sociale, da un elevato numero di figli da dover crescere, da violenze domestiche, da uno scarso livello di integrazione sociale e incertezze legate al futuro; possibili fattori protettivi, invece, sarebbero costituiti da buoni e forti legami familiari capaci di contenere l'impatto degli stressors sociali (tra tutti, un basso stato socio-economico) sulla salute mentale di migranti e minoranze etniche. Alcuni studi hanno evidenziato un'elevata tendenza alla manifestazione di disturbi somatici funzionali come equivalenti di sintomi ansioso-depressivi in migranti con recente storia di immigrazione, mentre una più vecchia storia di migrazione comporterebbe, come correlato del processo di acculturazione e di adattamento al paese di accoglienza, una maggiore espressività della sofferenza psichica secondo le tradizionali tipologie della cultura occidentale rappresentati da sintomi ansiosi e depressivi ${ }^{11}$. Diversi studi condotti a livello delle cure primarie evidenziano come riconoscimento e trattamento dei DMC sono inferiori tra le minoranze etniche rispetto ai nativi,

\footnotetext{
9 Morgan C., Mallett R., Hutchinson G., Bagalkote H., Morgan K., Fearon P., Dazzan P., Boydell J., McKenzie K., Harrison G., Murray R., Jones P., Craig T., Leff J., "Sample characteristics and compulsory admission: a report from the AESOP study", British Journal of Psychiatry, 186, 2005, pp. 281-289.

10 Nazroo JY., Edwards AC., Brown GW., "Gender differences in the onset of depression following a shared life event: a study of couples", Psychological Medecine, 27(1), 1997, pp. 9-19.

11 Nazroo JY., op. cit.; Bhugra D., Becker MA., "Migration, cultural bereavement and cultural identity", World Psychiatry, 4(1), 2005, pp. 18-24; Chaturvedi SK., Bhugra D., "The concept of neurosis in a cross-cultural
}

anche nei casi in cui $\mathrm{i}$ tassi di consultazione sono più elevati nei migranti rispetto alla popolazione autoctona $^{12}$. Fra i principali fattori legati ai problemi di identificazione e trattamento sono da segnalare la distanza culturale, la competenza culturale, caratteristiche psicopatologiche diverse (somatizzazione) e cultural idioms of distress.

\section{Psicosi e migrazione.}

La letteratura di settore ha ormai ampiamente evidenziato come i fattori di rischio per lo sviluppo di psicosi non sono solo individuali, ma anche di area, di neighbourhood, come, ad esempio, il vivere in aree urbane ${ }^{13}$. La genesi dei disturbi dello spettro psicotico percorre molteplici vie in cui il manifestarsi di sintomi conclamati di psicosi sarebbe il risultato dell'accumularsi durante il corso delle vita di stressor socio-ambientali che andrebbero a slatentizzare una preesistente vulnerabilità neuropsicologica ${ }^{14}$. Secondo il modello del sociosviluppo di Morgan, infatti, l'esposizione a traumi o ad avversità durante il corso della vita interagirebbe con una specifica suscettibilità genetica ed inciderebbe sullo sviluppo cerebrale nonché sulla sensibilità personale allo stress, generando uno stato di persistente vulnerabilità alla psicosi (costituita da bias sociali, esperienze simil-psicotiche e disturbi affettivi). Il contesto ambientale, pertanto, appare capace di interagire con la predisposizione genetica mediante il sistema epigenetico generando, nel corso della vita dell'individuo, una complessa ed

perspective", Current Opinion in Psychiatry, 20(1), 2007, pp, 47-51.

${ }_{12}$ Tarricone I., Migranti e competenza culturale nella consulenza psichiatrica in medicina generale, Bologna, martedì 16 febbraio 2010.

13 Murray RM., Dean K., "Schizofrenia e disturbi correlati", in Murray RM., Kendler KS., McGuffin P., Wessely S., Castle DJ., Fondamenti di Psichiatria, Edizioni Medico Scientifiche, Torino, 2011.

14 Morgan C., Charalambides M., Hutchinson G., Murray RM., "Migration, ethnicity, and psychosis: toward 
interconnessa rete di causazioni ${ }^{15}$. Risulta pertanto evidente come la migrazione rappresenti un'esperienza che si dipana nel corso della vita di un individuo in grado di assemblare e condensare vari fattori di rischio ambientali (isolamento sociale, stile di vita, capacità di relazionarsi, ambienti lavorativi ristretti, ecc.) responsabili di una pressione e di uno stress tali da poter condizionare in maniera significativa lo sviluppo di psicosi. Abbiamo infatti precedentemente sottolineato come lo status di migrante sia associato ad un aumentato rischio di disturbi mentali gravi, come le psicosi, rispetto allo status di nativo; in proposito la letteratura ha evidenziato un rischio di psicosi più di due volte superiore nei migranti, sia di prima che di seconda generazione, rispetto ai nativi ${ }^{16}$. Poiché nei paesi di origine dei migranti non si è riscontrato un aumento dell'incidenza delle psicosi, e quindi sarebbe da escludere che vi sia un maggior rischio geneticamente determinato nelle persone che provengono da tali paesi di sviluppare un disturbo psicotico, particolare attenzione è stata posta ai

a socio developmental model", Schizophrenia Bulletin 36, 4, 2010, pp. 655-664.

15 Shah J., Mizrahi R., McKenzie K., "The four dimensions: a model for the social aetiology of psychosis", British Journal of Psychiatric, 199, 1, 2011, pp. 11-14.

${ }^{16}$ Harrison G., Holton A., Neilson D., Owens D., Boot D., Cooper J., "Severe mental disorder in Afro-Caribbean patients: some social, demographic and service factors", Psychological Medicine, 19(3), 1989, pp. 683-696; CantorGraee E., Selten JP., "Schizophrenia and migration: a meta-analysis and review", American Journal of Psychiatric, 162, 2005, pp. 12-24; Morgan C., Charalambides M., Hutchinson G., Murray RM., "Migration, ethnicity, and psychosis: toward a socio developmental model", Schizophrenia Bulletin 36, 4, 2010, pp. 655-664; Tarricone I., Atti A.R., Salvatori F., Braca M., Ferrari S., Malmusi D., Berardi D., "Psychotic symptoms and general health in a socially disadvantaged migrant community in Bologna", International Journal of Social Psychiatry, 55, 2009, pp. 203-213; Tarricone I., Stivanello E., Ferrari S., Colombini N., Bolla E., Braca M., Giubbarelli C., Costantini C., Cazzamalli S., Mimmi S., Tedesco D., Menchetti M., Rigatelli M., Maso E., Balestrieri M., Vender S., Berardi D., "Migrant pathways to community mental health centres in Italy", International Journal of Social Psychiatry, 58, 2012, pp. 505-511. fattori ambientali nella genesi di tale disturbo. Infatti, numerosi studi, condotti soprattutto in ambiente nord europeo e anglosassone, hanno rilevato come i più importanti fattori causali siano collocati nella fase post-migratoria e, in particolare, risiedano nella difficoltà di integrazione nella società ospite, a causa dello svantaggio sociale, della discriminazione, del "mismatch" tra aspettative e realizzazione ${ }^{17}$. Pertanto, gli studi negli ultimi 20 anni si sono concentrati sui fattori ambientali che possono essere responsabili di tale "eccesso di psicosi". Tali fattori ambientali possono agire sia a livello individuale, che sociale e si possono schematicamente collocare nelle 3 fasi del processo migratorio $^{18}$ : durante la fase pre-migratoria (complicanze ostetriche dovute alle cattive condizioni igienico-sanitarie del paese di origine, fattori infettivi, deficit di vitamina $\mathrm{D}$, ecc.); durante la fase migratoria (trauma del viaggio, scarsa preparazione della migrazione, ecc.), durante la fase post-migratoria (discriminazione, disoccupazione, basso status socio-economico, razzismo, isolamento, "urbanizzazione" e densità etnica). Tali studi, condotti soprattutto in ambiente nord europeo e anglosassone, hanno rilevato come i più importanti fattori causali siano collocati nella fase post-migratoria ed, in particolare, risiedano nelle difficoltà di integrazione nella società ospite. Oltre a fattori che intervengono sul singolo individuo per determinarne la predisposizione alla psicosi, quali lo svantaggio sociale, la discriminazione, il "mismatch" tra aspettative e realizzazione, sembrano avere un

17 Morgan C., Charalambides M., Hutchinson G., Murray RM., "Migration, ethnicity, and psychosis: toward a socio developmental model", Schizophrenia Bulletin 36, 4, 2010, pp. 655-664; Murray RM., Dean K., "Schizofrenia e disturbi correlati", in Murray RM., Kendler KS., McGuffin P., Wessely S., Castle DJ., Fondamenti di Psichiatria, Edizioni Medico Scientifiche, Torino, 2011.

18 Bhugra D., Becker MA., "Migration, cultural bereavement and cultural identity", World Psychiatry, 4(1), 2005, pp. 18-24. 
ruolo rilevante fattori che agiscono a livello di area. Studi inglesi, infatti, hanno evidenziato che l'incidenza di schizofrenia è inferiore nelle zone dove gli White British e le minoranze etniche vivono in gruppi più coesi e meno frammentati ${ }^{19}$. Un'ipotesi è che il capitale sociale possa "mediare" tra fattori di rischio e psicosi; in accordo con le evidenze di letteratura ${ }^{20} \mathrm{i}$ più alti tassi di psicosi si avrebbero, quindi, nei contesti più disorganizzati delle città, non necessariamente nei più poveri. Resta da accertare, data l'attuale mancanza di studi al riguardo, il ruolo di fattori traumatici durante la fase migratoria, del "viaggio"dal paese di origine e a quello di approdo.

\section{Conclusioni.}

L'immigrazione rappresenta un fenomeno per certi sensi "ambiguo" in quanto se, da un lato, può ampliare l'opportunità di scelta esistenziale e di azione del soggetto, dall'altro sottopone l'individuo ad una grave esposizione a fattori di svantaggio sociale, con la possibilità di generare profonde crisi anche psicopatologiche. Confrontarsi con i migranti rappresenta oggi una priorità di salute mentale pubblica, oltre ad una importante occasione per comprendere i meccanismi di interazione fra fattori di vulnerabilità individuale e fattori di rischio ambientali coinvolti nell'eziopatogenesi dei disturbi psicotici $^{21}$.

19 Kirkbride JB., Fearon P., Morgan C., Dazzan P., Morgan K., Murray RM., Jones PB., "Neighbourhood variation in the incidence of psychotic disorders in Southeast London", Social Psychiatry and Psychiatric Epidemiology, 42(6), 2007, pp. 438-445.

20 Morgan C., Charalambides M., Hutchinson G., Murray RM., "Migration, ethnicity, and psychosis: toward a socio developmental model", Schizophrenia Bulletin 36, 4, 2010, pp. 655-664.

21 Murray RM., Dean K., "Schizofrenia e disturbi correlati", in Murray RM., Kendler KS., McGuffin P., Wessely S., Castle DJ., Fondamenti di Psichiatria, Edizioni Medico Scientifiche, Torino, 2011; Tarricone I., Cianconi P., Chierzi F. et al., "Comprendere le cause dell'esordio
Il notevole aumento di persone appartenenti a popolazioni diverse dal punto di vista etnico e culturale che si rivolgono ai servizi sanitari necessita che gli operatori della salute, ed in particolare quelli della salute mentale, siano in grado di rispondere alle esigenze di questa nuova fascia di utenti. Si impone, in particolare, la necessità di creare équipe dedicate multiprofessionali in grado di fornire valutazioni diagnostiche culturalmente orientate, appropriati interventi sociali finalizzati all'individuazione dei fattori di rischio specifici dello status di migrante e transculturali (choc culturale, difficoltà linguistiche, svantaggi sociali, ecc.), nonché approcci terapeutici definiti di Cognitive Behavioural Therapy, che mirino alla restrutturazione cognitiva delle esperienze legate alla discriminazione e al potenziamento della abilità sociali22. Per far questo è necessario che anche nel nostro paese, così come nel resto d'Europa, si intervenga a vari livelli, riducendo la difficoltà di accesso alle cure che caratterizza la popolazione migrante attraverso, innanzitutto, l'implemento dell'accesso alle cure primarie per migranti regolari e irregolari (ambulatori STP), lo sviluppo di servizi di consulenza e collegamento tra cure primarie, ambulatori STP e Centri di Salute mentale (CSM) e, non ultimo, implementando la competenza culturale nei CSM riguardo le problematiche e le specificità proprie della psichiatria transculturale, onde permettere una tempestiva e precisa valutazione

psicotico dall'ascolto della storia migratoria: il lavoro del Bologna Transcultural Psychiatric Team (BOTPT)", in Popolo R., Balbi A., Vinci G., Interventi precoci nelle psicosi, Alpes Italia srl, Roma 2013.

22 Burnett R., Mallett R., Bhugra D., Hutchinson G., Der G., Leff J., "The first contact of patients with schizophrenia with psychiatric services: social factors and pathways to care in a multiethnic population", Psychological Medicine, 29, 1999, pp. 475-483. 
della gravità di ogni singolo caso specialmente quelli all'esordio psicotico ${ }^{23}$.

\section{Bibliografia.}

- Bhugra D., Becker MA., "Migration, cultural bereavement and cultural identity", World Psychiatry, 4(1), 2005, pp. 18-24.

- Boydell J., van Os J., Mckenzie K. et al., "Incidence of schizophrenia in ethnic minorities in London: ecological study into interactions with environment", British Medical Journal, 323, 2001, pp. 1336-1338.

- Braca M., Berardi D., Mencacci E., Murri M.B., Mimmi S., Allegri F., Mazzi F., Menchetti M., Tarricone I., "Understanding psychopathology in migrants: A mixed categoricaldimensional approach", International Journal of Social Psychiatry, 2, June 2013.

- Bria P., Caroppo E., Salute mentale, migrazione e pluralismo culturale, Alpes Italia srl, Roma, 2008.

- Burnett R., Mallett R., Bhugra D., Hutchinson G., Der G., Leff J., "The first contact of patients with schizophrenia with psychiatric services: social factors and pathways to care in a multiethnic population", Psychological Medicine, 29, 1999, pp. 475-483.

- Cantor-Graee E., Selten JP., "Schizophrenia and migration: a meta-analysis and review", American Journal of Psychiatric, 162, 2005, pp. 1224.

- Caritas/Migrantes, Immigrazione: Dossier Statistico 2010, Edizioni IDOS, Roma, 2010.

- Chaturvedi SK., Bhugra D., "The concept of neurosis in a cross-cultural perspective", Current Opinion in Psychiatry, 20(1), 2007, pp, 47-51.

- Cole E., Leavey G., King M., Johnson-Sabine E., Hoar A. "Pathways to care for patients with a first episode of psychosis. A comparison of ethnic groups", British Journal of Psychiatry, 167, 1995, pp. 770-776.

- Harrison G., Holton A., Neilson D., Owens D., Boot D., Cooper J., "Severe mental disorder in Afro-Caribbean patients: some social, demographic and service factors", Psychological Medicine, 19(3), 1989, pp. 683-696.

\footnotetext{
${ }^{23}$ Norman RMG., Townsed L., Malla AK., "Duration untreated psychosis and cognitive functioning in first episode patients", British Journal of Psychiatry, 179, 2001, pp. 340-345; Walters P., Tylee A., Goldberg D., "La psichiatria nell'assistenza primaria", in Murray RM., Kendler KS., McGuffin P., Wessely S., Castle DJ., Fondamenti di Psichiatria, Edizioni Medico Scientifiche, Torino, 2011.
}

- Kirkbride JB., Fearon P., Morgan C., Dazzan P., Morgan K., Murray RM., Jones PB., "Neighbourhood variation in the incidence of psychotic disorders in Southeast London", Social Psychiatry and Psychiatric Epidemiology, 42(6), 2007, pp. 438-445.

- Merleau-Ponty M., Fenomenologia delle percezione,.Bompiani, Torino 2003.

- Morgan C., Charalambides M., Hutchinson G., Murray RM., "Migration, ethnicity, and psychosis: toward a socio developmental model", Schizophrenia Bulletin 36, 4, 2010, pp. 655-664.

- Morgan C., Mallett R., Hutchinson G., Bagalkote H., Morgan K., Fearon P., Dazzan P., Boydell J., McKenzie K., Harrison G., Murray R., Jones P., Craig T., Leff J., "Sample characteristics and compulsory admission: a report from the AESOP study", British Journal of Psychiatry, 186, 2005, pp. 281-289.

- Murray RM., Dean K., "Schizofrenia e disturbi correlati", in Murray RM., Kendler KS., McGuffin P., Wessely S., Castle DJ., Fondamenti di Psichiatria, Edizioni Medico Scientifiche, Torino, 2011.

- Nazroo JY., Edwards AC., Brown GW., "Gender differences in the onset of depression following a shared life event: a study of couples", Psychological Medecine, 27(1), 1997, pp. 9-19.

- Norman RMG., Townsed L., Malla AK., "Duration untreated psychosis and cognitive functioning in first episode patients", British Journal of Psychiatry, 179, 2001, pp. 340-345.

- Shah J., Mizrahi R., McKenzie K., "The four dimensions: a model for the social aetiology of psychosis", British Journal of Psychiatric, 199, 1, 2011, pp. 11-14.

- Sossi F., Migrare, Il Saggiatore, Roma, 2007.

- Tarricone I., Cianconi P., Chierzi F. et al., "Comprendere le cause dell'esordio psicotico dall'ascolto della storia migratoria: il lavoro del Bologna Transcultural Psychiatric Team (BOTPT)", in Popolo R., Balbi A., Vinci G., Interventi precoci nelle psicosi, Alpes Italia srl, Roma 2013.

- Tarricone I., Migranti e competenza culturale nella consulenza psichiatrica in medicina generale, Bologna, martedì 16 febbraio 2010.

- Tarricone I., Atti A.R., Salvatori F., Braca M., Ferrari S., Malmusi D., Berardi D., "Psychotic symptoms and general health in a socially disadvantaged migrant community in Bologna”, International Journal of Social Psychiatry, 55, 2009, pp. 203-213.

- Tarricone I., Stivanello E., Ferrari S., Colombini N., Bolla E., Braca M., Giubbarelli C., 
Costantini C., Cazzamalli S., Mimmi S., Tedesco D., Menchetti M., Rigatelli M., Maso E., Balestrieri M., Vender S., Berardi D., "Migrant pathways to community mental health centres in Italy", International Journal of Social Psychiatry, 58, 2012, pp. 505-511.
- Walters P., Tylee A., Goldberg D., "La psichiatria nell'assistenza primaria", in Murray RM., Kendler KS., McGuffin P., Wessely S., Castle DJ., Fondamenti di Psichiatria, Edizioni Medico Scientifiche, Torino, 2011. 